\title{
Reflexivity of the translation-dilation algebras on $L^{2}(\mathbb{R})$
}

\author{
R. H. Levene and S. C. Power
}

August 16, 2018

\begin{abstract}
The hyperbolic algebra $\mathcal{A}_{h}$, studied recently by Katavolos and Power [5], is the weak star closed operator algebra on $L^{2}(\mathbb{R})$ generated by $H^{\infty}(\mathbb{R})$, as multiplication operators, and by the dilation operators $V_{t}, t \geq 0$, given by $V_{t} f(x)=e^{t / 2} f\left(e^{t} x\right)$. We show that $\mathcal{A}_{h}$ is a reflexive operator algebra and that the four dimensional manifold Lat $\mathcal{A}_{h}$ (with the natural topology) is the reflexive hull of a natural two dimensional subspace.
\end{abstract}

\section{Introduction}

We resolve some problems posed in [5] for the following nonselfadjoint operator algebra $\mathcal{A}_{h}$. Let $M_{\lambda}$ and $V_{t}$ be the multiplication and dilation operators on $L^{2}(\mathbb{R})$ given by

$$
M_{\lambda} f(x)=e^{i \lambda x} f(x), \quad V_{t} f(x)=e^{t / 2} f\left(e^{t} x\right)
$$

and let $\mathcal{A}_{h}$, the so-called hyperbolic algebra, be the weak-star closed operator algebra generated by $\left\{M_{\lambda}, V_{t} \mid \lambda, t \geq 0\right\}$. We can regard $\mathcal{A}_{h}$ as a doubly nonselfadjoint algebra in the sense that it is generated by the two copies of $H^{\infty}(\mathbb{R})$ generated by these unitary semigroups separately. Under conjugation by the Fourier-Plancherel transform the generators correspond to the translations $D_{\lambda} f(x)=f(x-\lambda)$ and dilations (for $t \leq 0$ ) and for this reason we refer to $\mathcal{A}_{h}$ as a translation-dilation algebra. The weak star closed translation-multiplication algebra, $\mathcal{A}_{p}$ say, generated by $\left\{M_{\lambda}, D_{\mu} \mid \lambda, \mu \geq 0\right\}$, is also doubly nonselfadjoint and was analysed in [4] and [8]. The algebras $\mathcal{A}_{p}$ and $\mathcal{A}_{h}$ have similar properties and may be viewed as fundamental examples of Lie semigroup algebras. By this we mean that

2000 Mathematics Subject Classification. Primary 46K50.

Keywords: reflexive algebra, translation invariant, Lie semigroup, hyperbolic algebra. The first author is supported by an EPSRC grant. 
they are weak star closed operator algebras generated by the image of a Lie semigroup under a unitary representation of the ambient Lie group. Indeed $\mathcal{A}_{h}$ arises in this way from the unitary representation of the $a x+b$ group given by

$$
\left(\begin{array}{ll}
a & b \\
0 & 1
\end{array}\right) \mapsto M_{b} V_{\log a}, \quad a \neq 0, b \in \mathbb{R},
$$

together with the Lie semigroup for $a \geq 1, b \geq 0$.

It is a fundamental result of Sarason [9] that $H^{\infty}(\mathbb{R})$ acting as a multiplication algebra on $H^{2}(\mathbb{R})$ or $L^{2}(\mathbb{R})$ is a reflexive operator algebra. This means that the invariant subspace lattice $\mathcal{L}=$ Lat $\mathcal{A}$ of the operator algebra $\mathcal{A}$ is such that

$$
\mathcal{A}=\operatorname{Alg} \mathcal{L}=\{A \mid A K \subseteq K \text { for all } K \in \mathcal{L}\} .
$$

Since $\operatorname{Alg} \mathcal{L}$ is the maximal operator algebra with lattice $\mathcal{L}$ (and hence reflexive) it is of particular interest to determine reflexivity for operator algebras specified by generators, and indeed, such a determination can be related to subtle problems of synthesis. This is evident in Arveson's delicate analysis of operator algebra synthesis and the density of pseudo-integral operators in CSL algebras (see [1] and also Froelich [3]). See also the slice map analysis of the tensor product formula (and its failure) for various reflexive algebras in [6], the analysis of reflexivity for subnormal operators in [10, and the counterexamples of Wogen and Larson in [1] and [7.

In 4. A. Katavolos and the second author showed that the translationmultiplication algebra on $L^{2}(\mathbb{R})$ is reflexive by identifying it with the (evidently) reflexive algebra

$$
\mathcal{A}_{F B}=\operatorname{Alg}\left(\mathcal{N}_{a} \cup \mathcal{N}_{v}\right)=\left(\operatorname{Alg} \mathcal{N}_{a}\right) \cap\left(\operatorname{Alg} \mathcal{N}_{v}\right)
$$

where $\mathcal{N}_{a}=\left\{e^{i \lambda x} H^{2}(\mathbb{R}) \mid \lambda \in \mathbb{R}\right\}$ and $\mathcal{N}_{v}=\left\{L^{2}[t, \infty] \mid-\infty \leq t \leq+\infty\right\}$ are the so-called analytic nest and the Volterra nest respectively. The lattice $\mathcal{N}_{a} \cup \mathcal{N}_{v}$, which we call the Fourier binest, is homeomorphic to the unit circle when endowed with the natural topology, the relative weak operator topology on the lattice of associated orthogonal projections. Whilst this lattice provides the obvious subspaces in $\mathcal{L}_{p}=$ Lat $\mathcal{A}_{p}$, the lattice $\mathcal{L}_{p}$ turns out to be bigger and is homeomorphic to the unit disc, with the Fourier binest as topological boundary. That is, $\mathcal{L}_{p}$ is the reflexive hull of $\mathcal{N}_{a} \cup \mathcal{N}_{v}$;

$$
\mathcal{L}_{p}=\operatorname{Lat} \operatorname{Alg}\left(\mathcal{N}_{a} \cup \mathcal{N}_{v}\right) .
$$

The algebras $\mathcal{A}_{p}$ and $\mathcal{A}_{h}$ share several features over and above being doubly nonselfadjoint. They are antisymmetric $\left(\mathcal{A} \cap \mathcal{A}^{*}=\mathbb{C} I\right)$ and they contain no non-trivial finite rank operators. Both these facts rule out many of the basic techniques available for the study of nest algebras (see [2]). On the other hand, $\mathcal{A}_{p}$ and $\mathcal{A}_{h}$ are not unitarily equivalent (nor isometrically 
isomorphic) and $\mathcal{L}_{h}=$ Lat $\mathcal{A}_{h}$ is a 4-dimensional Euclidean manifold which we indicate in Section 4. The main results of the present paper show that $\mathcal{A}_{h}$ is a reflexive operator algebra and, in parallel with the comments above for $\mathcal{A}_{p}$, that $\mathcal{L}_{h}$ is the reflexive hull of the sublattice

$$
\mathcal{L}_{M} \cup \mathcal{L}_{S}
$$

where

$$
\mathcal{L}_{M}=\left\{L^{2}[-a, b] \mid a, b \in[0, \infty]\right\}
$$

and

$$
\mathcal{L}_{S}=\left\{|x|^{i s} H^{2}(\mathbb{R}) \mid s \in \mathbb{R}\right\} \cup\left\{0, L^{2}(\mathbb{R})\right\} .
$$

Furthermore we obtain an explicit characterisation of the Hilbert-Schmidt operators in $\mathcal{A}_{h}$, and in fact this is the key to the proof of reflexivity.

\section{Hilbert-Schmidt operators in $\operatorname{Alg}\left(\mathcal{L}_{M} \cup \mathcal{L}_{S}\right)$}

We write $\mathcal{C}_{2}$ for the set of Hilbert-Schmidt operators on $L^{2}(\mathbb{R})$, so that $\mathcal{C}_{2}=\left\{\operatorname{Int} k \mid k \in L^{2}\left(\mathbb{R}^{2}\right)\right\}$ where

$$
(\operatorname{Int} k) f(x)=\int k(x, y) f(y) d y .
$$

The following result gives a necessary support condition for Int $k$ to be in $\operatorname{Alg} \mathcal{L}_{M}$, and is simply proven. It is used several times without reference when changing variables in what follows.

Lemma 1. Let Int $k \in \mathcal{C}_{2} \cap \operatorname{Alg} \mathcal{L}_{M}$. Then

$$
\operatorname{supp} k \subseteq\left\{(x, y) \in \mathbb{R}^{2} \mid x y \geq 0 \text { and }|y| \geq|x|\right\} .
$$

The lemma shows that a Hilbert-Schmidt operator which leaves invariant all the subspaces $L^{2}[-a, b]$ of $\mathcal{L}_{M}$ has kernel function $k(x, y)$ supported in the cone bounded by the $y$-axis and the line $y=x$. We now investigate the further constraints on $k(x, y)$ in order that Int $k$ should also leave invariant all the subspaces $|x|^{i s} H^{2}(\mathbb{R})$ of the set $\mathcal{L}_{S}$. We shall show that the condition corresponds to the radially restricted functions $x \mapsto k\left(x, e^{t} x\right)$ lying in an appropriate function space for almost every $t$. We begin by defining this space, $V$.

Let $\mathbb{H}^{+}$denote the open upper half-plane in $\mathbb{C}$ and $\mathbb{H}^{-}$the open lower half-plane. Let $\mathbb{H}_{\mathbb{Q}}^{-}$be the set of points in $\mathbb{H}^{-}$with rational real and complex parts. For $v, w \in \mathbb{H}^{-}$, set

$$
\tilde{r}_{v, w}(z)=\frac{1}{(z-v)(z-w)}
$$


Then $\tilde{r}_{v, w}$ is analytic in $\mathbb{H}^{+}$; let $r_{v, w}$ be the boundary value function of $\tilde{r}_{v, w}$ on the real line.

The density assertion of the next lemma follows routinely from Cauchy's theorem and the definition of $H^{2}(\mathbb{R})$. It will be useful for us in the proof of Proposition 6.

Lemma 2. The set $\mathcal{R}_{\mathbb{Q}}=\left\{r_{v, w} \mid v, w \in \mathbb{H}_{\mathbb{Q}}^{-}\right\}$has dense linear span in $H^{2}(\mathbb{R})$.

For $\alpha \in \mathbb{R}$ and $z \in \mathbb{H}^{+} \cup \mathbb{R} \backslash\{0\}$, let

$$
\tilde{p}_{\alpha}(z)=\exp (\alpha \log z)
$$

where $\log z=\log |z|+i \operatorname{Arg} z$ and $\operatorname{Arg} z$ is the $\operatorname{argument}$ function taking values in $[0, \pi]$. Then $\tilde{p}_{\alpha}$ is analytic in $\mathbb{H}^{+}$. Let $p_{\alpha}$ be the restriction of $\tilde{p}_{\alpha}$ to $\mathbb{R} \backslash\{0\}$ and let $p=p_{1 / 2}$ and $q=p_{-1 / 2}$. Note that $\overline{p(x)}=\operatorname{sgn}(x) p(x)$ and $\overline{q(x)}=\operatorname{sgn}(x) q(x)$ where $\operatorname{sgn}(x)=x /|x|$ for $x \neq 0$.

Now define the space $V$ to be the closed linear span in $L^{2}(|x| d x)$ of the set $\left\{\bar{q} r_{v, w} \mid v, w \in \mathbb{H}_{\mathbb{Q}}^{-}\right\}$. Observe that the unitary $M_{\bar{p}}: L^{2}(|x| d x) \rightarrow L^{2}(\mathbb{R})$ restricts to a unitary map $V \rightarrow H^{2}(\mathbb{R})$, and $M_{\bar{p}}^{*}=M_{\bar{q}}$. This shows that, writing cl-span $A$ for the closed linear span of $A$, we have

$$
\begin{aligned}
V^{\perp} & =M_{\bar{p}}^{*}\left(M_{\bar{p}} V\right)^{\perp} \\
& =M_{\bar{q}}\left(H^{2}(\mathbb{R})\right)^{\perp} \\
& =M_{\bar{q}} \overline{H^{2}(\mathbb{R})} \\
& =M_{\bar{q}} \operatorname{cl}-\operatorname{span}\left\{\overline{r_{v, w}} \mid v, w \in \mathbb{H}_{\mathbb{Q}}^{-}\right\} \\
& =M_{\text {sgn }} \operatorname{cl}-\operatorname{span}\left\{q \overline{r_{v, w}} \mid v, w \in \mathbb{H}_{\mathbb{Q}}^{-}\right\} \\
& =M_{\text {sgn }} \bar{V} .
\end{aligned}
$$

Henceforth let $W=L^{2}\left(\mathbb{R}_{+}, e^{t} d t\right)$. We will write $\|\cdot\|_{V}$ and $\|\cdot\|_{W}$ for norms taken in the spaces $L^{2}(|x| d x)$ and $L^{2}\left(e^{t} d t\right)$ respectively.

The next lemma is elementary.

Lemma 3. Let $\mu$ and $\nu$ be measures on $\mathbb{R}$, and let $M$ and $N$ be closed subspaces of $L^{2}(\mu)$ and $L^{2}(\nu)$ respectively. Let $f \in L^{2}(\mu \times \nu)$, and suppose that for almost every $y, x \mapsto f(x, y) \in M$, and for almost every $x, y \mapsto$ $f(x, y) \in N$. Then $f \in M \otimes N$, the tensor product Hilbert space.

We now obtain a necessary condition on the kernel functions $k(x, y)$ of operators in $\operatorname{Alg}\left(\mathcal{L}_{M} \cup \mathcal{L}_{S}\right)$. Here and elsewhere we make the natural identification between the tensor product $M \otimes N$ and the closed linear span of $\{m(x) n(y) \mid m \in M, n \in N\}$ in $L^{2}(\mu \times \nu)$.

Proposition 4. Let Int $k$ be a Hilbert-Schmidt operator leaving invariant each of the subspaces $L^{2}[-a, b], a, b \geq 0$ and $|x|^{i s} H^{2}(\mathbb{R}), s \in \mathbb{R}$. Then the function $(x, t) \mapsto k\left(x, e^{t} x\right)$ is in the tensor product Hilbert space $V \otimes W$. 
Proof. We aim to apply Lemma 3. Firstly, for almost every $x, t \mapsto k\left(x, e^{t} x\right) \in$ $W$, since

$$
\iint|k(x, y)|^{2} d x d y=\iint\left|k\left(x, e^{t} x\right)\right|^{2} e^{t}|x| d x d t<\infty .
$$

This also shows that for almost every $t, x \mapsto k\left(x, e^{t} x\right)$ is in $L^{2}(|x| d x)$. It remains to show that this function lies in $V$.

Let $X=\operatorname{Int} k$. Then for every $s \in \mathbb{R}$ we have $X|x|^{i s} H^{2}(\mathbb{R}) \subseteq|x|^{i s} H^{2}(\mathbb{R})$, so for every $h_{1}$ and $h_{2}$ in $H^{2}(\mathbb{R})$,

$$
\begin{aligned}
0 & =\left\langle X|x|^{i s} h_{1},|x|^{i s} \overline{h_{2}}\right\rangle \\
& =\int\left[\int k(x, y)|y|^{i s} h_{1}(y) d y\right]|x|^{-i s} h_{2}(x) d x .
\end{aligned}
$$

Now using Lemma 1 we make the change of variables $y=e^{t} x$ to get

$$
0=\iint e^{t / 2}|x| k\left(x, e^{t} x\right) e^{i s t}\left(V_{t} h_{1}\right)(x) h_{2}(x) d t d x .
$$

Now

$$
\begin{aligned}
\iint\left|e^{t / 2} x k\left(x, e^{t} x\right)\left(h_{2} V_{t} h_{1}\right)(x)\right| d x d t & =\iint\left|k(x, y) h_{2}(x) h_{1}(y)\right| d x d y \\
& =\left\langle(\operatorname{Int}|k|)\left|h_{1}\right|,\left|h_{2}\right|\right\rangle<\infty,
\end{aligned}
$$

so the integrand in (10) is in $L^{1}(d t \times d x)$ and we can apply Fubini's theorem to get

$$
0=\int\left\{\int e^{t / 2}|x| k\left(x, e^{t} x\right)\left(h_{2} V_{t} h_{1}\right)(x) d x\right\} e^{i s t} d t .
$$

Let $T(t)$ be the expression in curly brackets. By virtue of (2) $T$ is in $L^{1}(\mathbb{R})$ and so (3) can be interpreted as saying that the Fourier transform of the $L^{1}$ function $T$ is zero. Hence for almost every $t, T(t)=0$.

For fixed $t$, as $h_{1}$ and $h_{2}$ run over $H^{2}(\mathbb{R}), h_{2} V_{t} h_{1}$ runs over $H^{1}(\mathbb{R})$, so we can pick $h_{1}$ and $h_{2}$ such that $h_{2} V_{t} h_{1}$ runs over a countable dense subset of $H^{2}(\mathbb{R})$. Since $T=0$, for almost every $t$ we have

$$
\int k\left(x, e^{t} x\right) f(x)|x| d x=0
$$

for every $f \in H^{2}(\mathbb{R})$. We can now approximate again: since $q r_{v, w}$ is in $H^{2}(\mathbb{R})$ for each $v, w \in \mathbb{H}^{-}$, for almost every $t$ we have

$$
0=\int k\left(x, e^{t} x\right) q(x) r_{v, w}(x)|x| d x=\left\langle x \mapsto k\left(x, e^{t} x\right), \overline{q r_{v, w}}\right\rangle_{V}
$$

for each $v, w \in \mathbb{H}_{\mathbb{Q}}^{-}$. But $\left\{\overline{q r_{v, w}} \mid v, w \in \mathbb{H}_{\mathbb{Q}}^{-}\right\}$is countable and has $\|\cdot\|_{V^{-}}$-dense linear span in $V^{\perp}$, so we conclude that for almost every $t, x \mapsto k\left(x, e^{t} x\right)$ is in $V$.

Lemma 1 and a simple computation show that for almost every $x, t \mapsto$ $k\left(x, e^{t} x\right)$ is in $W$. Now Lemma 3 ensures that $k\left(x, e^{t} x\right)$ is in $V \otimes W$. 


\section{Hilbert-Schmidt operators in $\mathcal{A}_{h}$}

We have the elementary inclusions

$$
\mathcal{A}_{h} \subseteq \operatorname{Alg}\left(\operatorname{Lat} \mathcal{A}_{h}\right) \subseteq \operatorname{Alg}\left(\mathcal{L}_{M} \cup \mathcal{L}_{S}\right)
$$

To show that we in fact have equalities, and in particular that $\mathcal{A}_{h}$ is reflexive, we shall establish two facts. Firstly, that the necessary condition of Proposition 4 is in fact sufficient for Int $k$ to belong to $\mathcal{A}_{h}$. Thus, $\mathcal{A}_{h}$ and $\operatorname{Alg}\left(\mathcal{L}_{M} \cup \mathcal{L}_{S}\right)$ will contain the same Hilbert-Schmidt operators. Secondly we show that for each algebra the Hilbert-Schmidt operators are dense for the weak star topology.

Lemma 5. Let $\varphi \in W$ have compact support. Then for $f \in L^{2}(\mathbb{R})$ with compact support and almost every $x \in \mathbb{R}$, the integral

$$
\tilde{V}_{\varphi} f(x)=\int e^{t / 2} \varphi(t) V_{t} f(x) d t
$$

converges, and $\tilde{V}_{\varphi}$ extends to a bounded operator $V_{\varphi} \in \mathcal{L}\left(L^{2}(\mathbb{R})\right)$. Moreover, $V_{\varphi} \in \mathcal{A}_{h}$.

Proof. Checking that $\tilde{V}_{\varphi} f(x)$ converges for almost every $x$ when $f$ is compactly supported is easily done. Let $f$ and $g$ be in $L^{2}(\mathbb{R})$. Then

$$
\begin{aligned}
\left|\iint e^{t / 2} \varphi(t) V_{t} f(x) \overline{g(x)} d x d t\right| & \leq \int\left|e^{t / 2} \varphi(t)\left\langle V_{t} f, g\right\rangle\right| d t \\
& \leq\|f\|\|g\|\|\varphi\|_{L^{1}\left(e^{t / 2} d t\right)} .
\end{aligned}
$$

Since $\varphi$ is compactly supported and in $W$, it is in $L^{1}\left(e^{t / 2} d t\right)$ and so the sesquilinear form

$$
\langle f, g\rangle_{\varphi}=\iint e^{t / 2} \varphi(t) V_{t} f(x) \overline{g(x)} d x d t
$$

is bounded and defines a bounded operator $V_{\varphi}$ extending $\tilde{V}_{\varphi}$.

To show that $V_{\varphi} \in \mathcal{A}_{h}$, without loss of generality we can assume that the support of $\varphi$ is contained in the interval $[0,1]$. For positive integers $m<n$, let

$$
\alpha_{n, m}=\int_{m / n}^{(m+1) / n} e^{s / 2} \varphi(s) d s,
$$

and let $T_{n}=\sum_{m=0}^{n-1} \alpha_{n, m} V_{m / n}$. Then $T_{n} \in \mathcal{A}_{h}$ for every $n$. We claim that 
$T_{n} \rightarrow V_{\varphi}$ in WOT. For if $f, g \in L^{2}(\mathbb{R})$, then

$$
\begin{aligned}
\left\langle\left(V_{\varphi}-T_{n}\right) f, g\right\rangle & =\int \overline{g(x)}\left(\int e^{t / 2} \varphi(t)\left(V_{t} f\right)(x) d t-\sum_{m=0}^{n-1} \alpha_{n, m}\left(V_{m / n} f\right)(x)\right) d x \\
& =\int \overline{g(x)} \sum_{m=0}^{n-1} \int_{m / n}^{(m+1) / n} e^{t / 2} \varphi(t)\left(\left(V_{t} f\right)(x)-\left(V_{m / n} f\right)(x)\right) d t d x \\
& =\int e^{t / 2} \varphi(t)\left(\left\langle V_{t} f, g\right\rangle-\left\langle V_{m(n, t) / n} f, g\right\rangle\right) d t
\end{aligned}
$$

where $m(n, t)=\lfloor n t\rfloor$. Now for any $t,\left|\left\langle V_{t} f, g\right\rangle\right| \leq\|f\|\|g\|$, and $e^{t / 2} \varphi(t)$, being a compactly supported element of $L^{2}(\mathbb{R})$, is in $L^{1}(\mathbb{R})$. Moreover, $V_{m(n, t) / n} \rightarrow V_{t}$ in WOT as $n \rightarrow \infty$. So by dominated convergence, $\left\langle\left(V_{\varphi}-\right.\right.$ $\left.\left.T_{n}\right) f, g\right\rangle \rightarrow 0$ as $n \rightarrow \infty$. For every $n,\left\|T_{n}\right\| \leq\|\varphi\|_{L^{1}\left(e^{t} d t\right)}$, so $\left(T_{n}\right)$ is a bounded sequence in $\mathcal{L}\left(L^{2}(\mathbb{R})\right)$. The $\mathrm{w}^{*}$-topology and WOT agree on bounded sets; since $\mathcal{A}_{h}$ is $\mathrm{w}^{*}$-closed, this shows that $V_{\varphi} \in \mathcal{A}_{h}$.

Given a function $F: \mathbb{R} \times \mathbb{R}_{+} \rightarrow \mathbb{C}$, we define the function $k_{F}: \mathbb{R}^{2} \rightarrow \mathbb{C}$ by

$$
k_{F}(x, y)=\left\{\begin{array}{ll}
F(x, \log (y / x)) & \text { if } x y>0 \\
0 & \text { otherwise }
\end{array} .\right.
$$

We also write $k_{h, \varphi}=k_{h(x) \varphi(t)}$ when $h: \mathbb{R} \rightarrow \mathbb{C}$ and $\varphi: \mathbb{R}_{+} \rightarrow \mathbb{C}$. Note that this means that $k_{h, \varphi}\left(x, e^{t} x\right)=h(x) \varphi(t)$ for $t>0$.

Proposition 6. Let $h \in V$ and $\varphi \in W$. Then $\operatorname{Int} k_{h, \varphi} \in \mathcal{A}_{h} \cap \mathcal{C}_{2}$.

Proof. Let $k=k_{h, \varphi}$. Since

$$
\begin{aligned}
\|k\|_{L^{2}\left(\mathbb{R}^{2}\right)}^{2} & =\iint|k(x, y)|^{2} d x d y \\
& =\int|\varphi(t)|^{2} e^{t} d t \cdot \int|h(x)|^{2}|x| d x \\
& =\|\varphi\|_{W}^{2}\|h\|_{V}^{2},
\end{aligned}
$$

we have $k \in L^{2}\left(\mathbb{R}^{2}\right)$ and so Int $k \in \mathcal{C}_{2}$. Since $\|k\|_{L^{2}\left(\mathbb{R}^{2}\right)}=\|\operatorname{Int} k\|_{\mathcal{C}_{2}}$, (5) also shows that if $h_{n} \rightarrow h$ in $\|\cdot\|_{V}$, then the operators Int $k_{h_{n}, \varphi}$ converge to Int $k$ in Hilbert-Schmidt norm, and so in operator norm. Since $\mathcal{A}_{h}$ is closed in operator norm, by the definition of $V$ we may assume that $h=\bar{q} r_{v, w}$ for some $v, w \in \mathbb{H}_{\mathbb{Q}}^{-}$. Also, if $\varphi$ has compact support, then

$$
\begin{aligned}
(\operatorname{Int} k) f(x) & =\int_{\{y \mid x y>0\}} h(x) \varphi(\log (y / x)) f(y) d y \\
& =|x| h(x) \int e^{t / 2} \varphi(t) V_{t} f(x) d t \\
& =M_{g} V_{\varphi} f(x),
\end{aligned}
$$


where $g(x)=|x| h(x)=p(x) r_{v, w}(x) \in H^{\infty}(\mathbb{R})$, as is easily verified. So Int $k=M_{g} V_{\varphi}$. Now $M_{g} \in \mathcal{A}_{h}$ since $g \in H^{\infty}(\mathbb{R})$. By Lemma $V_{\varphi} \in \mathcal{A}_{h}$ too, so their product Int $k$ is also in $\mathcal{A}_{h}$.

If $\varphi$ is not compactly supported, we can simply restrict $\varphi$ to compact subsets of $\mathbb{R}_{+}$to get a sequence of Hilbert-Schmidt operators in $\mathcal{A}_{h}$ that converges in Hilbert-Schmidt norm to Int $k$ by (5) again, and so in operator norm. So Int $k$ is in $\mathcal{A}_{h} \cap \mathcal{C}_{2}$ as desired.

Corollary 7. $\mathcal{A}_{h} \cap \mathcal{C}_{2}=\operatorname{Alg}\left(\mathcal{L}_{M} \cup \mathcal{L}_{S}\right) \cap \mathcal{C}_{2}=\left\{\operatorname{Int} k_{F} \mid F \in V \otimes W\right\}$.

Proof. Let $F \in V \otimes W$; then $F=\sum \lambda_{i} h_{i} \otimes \varphi_{i}$ with convergence in $L^{2}(|x| d x \times$ $\left.e^{t} d t\right)$. So Int $k_{F}=\sum \lambda_{i}$ Int $k_{h_{i}, \varphi_{i}}$ with convergence in Hilbert-Schmidt norm. By Proposition [6, each Int $k_{h_{i}, \varphi_{i}}$ is in $\mathcal{A}_{h} \cap \mathcal{C}_{2}$, so Int $k_{F}$ is too.

Conversely, if Int $k \in \operatorname{Alg}\left(\mathcal{L}_{M} \cup \mathcal{L}_{S}\right) \cap \mathcal{C}_{2} \supseteq \mathcal{A}_{h} \cap \mathcal{C}_{2}$ then Proposition 4 implies that $k=k_{F}$ for some $F \in V \otimes W$ and the corollary follows.

Proposition 8. $\mathcal{A}_{h} \cap \mathcal{C}_{2}$ contains a bounded approximate identity-that is, a sequence that is bounded in operator norm and converges in the strong operator topology to the identity.

Proof. Let

$$
h_{n}(x)=\frac{-n^{2} \operatorname{sgn}(x)}{\left(x+n^{-1} i\right)(x+n i)^{2}}, \quad \varphi_{n}(t)=n e^{-t / 2} \chi_{\left[0, n^{-1}\right]}(t)
$$

and let $g_{n}(x)=|x| h_{n}(x)$. Then $\left|g_{n}(x)\right| \leq 1$ for each real $x$ and $g_{n}(x) \rightarrow 1$ uniformly on compact sets, so $M_{g_{n}} \rightarrow I$ in SOT. Since $\bar{p} h_{n} \in H^{2}(\mathbb{R})$, we have $h_{n} \in V$. Moreover, $\varphi_{n} \in W$, and a calculation using Jensen's inequality shows that $\left\|V_{\varphi_{n}}\right\| \leq 1$ for each $n$. If we take $f \in L^{2}(\mathbb{R})$ continuous with compact support then by dominated convergence,

$$
\left\|V_{\varphi_{n}} f-f\right\|^{2}=\int\left|\int_{0}^{1 / n} n V_{t} f(x) d t-f(x)\right|^{2} d x \rightarrow 0 \text { as } n \rightarrow \infty .
$$

Since such functions are dense in $L^{2}(\mathbb{R}), V_{\varphi_{n}} \rightarrow I$ boundedly in SOT. Let $k_{n}=k_{h_{n}, \varphi_{n}}$. Then, exactly as in the proof of Proposition [6. Int $k_{n}=$ $M_{g_{n}} V_{\varphi_{n}} \in \mathcal{A}_{h} \cap \mathcal{C}_{2}$ and so Int $k_{n} \rightarrow I$ in SOT.

Remark. The bounded approximate identity above is considerably simpler than that obtained in [5].

\section{Reflexivity}

In [5], A. Katavolos and the second author showed that the lattice of invariant subspaces for $\mathcal{A}_{h}$ is

$$
\mathcal{L}_{h}=\operatorname{Lat} \mathcal{A}_{h}=\left(\bigcup_{s \in \mathbb{R},|\theta|=1} \mathcal{L}_{s, \theta}\right) \cup \mathcal{L}_{M}
$$


where

$$
\mathcal{L}_{s, \theta}=\left\{u_{\theta}(x)|x|^{i s} e^{i\left(\lambda x+\mu x^{-1}\right)} H^{2}(\mathbb{R}) \mid \lambda, \mu \geq 0\right\} .
$$

Here $u_{\theta}(x)$ is the function taking the value 1 for $x>0$ and $\theta$ for $x<0$. Also it was shown that with the natural topology $\mathcal{L}_{h}$ is connected and is a 4-dimensional Euclidean manifold.

Theorem 9. The hyperbolic algebra $\mathcal{A}_{h}$ is reflexive.

Proof. Take the sequence $X_{n}$ in $\mathcal{A}_{h} \cap \mathcal{C}_{2}$ from Proposition 8 such that $\left\|X_{n}\right\| \leq$ 1 for every $n$ and $X_{n} \rightarrow I$ strongly. If $T$ is an operator in $\mathcal{A}_{h}$, then $T$ is the SOT limit of the operators $T X_{n} \in \mathcal{A}_{h} \cap \mathcal{C}_{2}$. Similarly, if $S$ is an operator in $\operatorname{Alg} \mathcal{L}_{h}$, then $S$ is the SOT limit of the operators $S X_{n} \in \operatorname{Alg}\left(\mathcal{L}_{M} \cup \mathcal{L}_{S}\right) \cap \mathcal{C}_{2}$. Since $\mathcal{A}_{h}$ and $\operatorname{Alg}\left(\mathcal{L}_{M} \cup \mathcal{L}_{S}\right)$ are both strongly closed and by the results of Section 3 they contain the same Hilbert-Schmidt operators, upon taking strong operator topology closures, we see that

$$
\mathcal{A}_{h}=\operatorname{SOT}-\operatorname{cl}\left(\mathcal{A}_{h} \cap \mathcal{C}_{2}\right)=\operatorname{SOT}-\operatorname{cl}\left(\operatorname{Alg}\left(\mathcal{L}_{M} \cup \mathcal{L}_{S}\right) \cap \mathcal{C}_{2}\right)=\operatorname{Alg}\left(\mathcal{L}_{M} \cup \mathcal{L}_{S}\right) .
$$

So the inclusions in (4) are equalities and $\mathcal{A}_{h}$ is reflexive.

We now obtain some consequences for sublattices of $\mathcal{L}_{h}$. Note that part (ii) of the next theorem is analogous to the fact that the lattice $\mathcal{L}_{p}$ of the translation-multiplication algebra $\mathcal{A}_{F B}$ is the reflexive hull of the Fourier binest $\mathcal{N}_{a} \cup \mathcal{N}_{v}$.

Theorem 10. Let $\mathcal{L}_{M}, \mathcal{L}_{V}$ and $\mathcal{L}_{S}$ be the subspace lattices

$$
\begin{aligned}
\mathcal{L}_{M} & =\left\{L^{2}[-a, b] \mid a, b \in[0, \infty]\right\}, \\
\mathcal{L}_{V} & =\left\{u_{\theta}(x)|x|^{i s} H^{2}(\mathbb{R}) \mid \theta \in S^{1}, s \in \mathbb{R}\right\} \cup\left\{0, L^{2}(\mathbb{R})\right\}, \\
\mathcal{L}_{S} & =\left\{|x|^{i s} H^{2}(\mathbb{R}) \mid s \in \mathbb{R}\right\} \cup\left\{0, L^{2}(\mathbb{R})\right\} .
\end{aligned}
$$

(i) The lattice $\mathcal{L}_{M}$ (respectively $\mathcal{L}_{V}$ ) is the set of subspaces of $\mathcal{L}_{h}$ which are reducing subspaces for the semigroup $\left\{M_{\lambda} \mid \lambda \in \mathbb{R}_{+}\right\}$(respectively $\left\{V_{t} \mid\right.$ $\left.t \in \mathbb{R}_{+}\right\}$).

(ii) $\mathcal{L}_{h}$ is the reflexive hull of $\mathcal{L}_{M} \cup \mathcal{L}_{V}$.

(iii) $\mathcal{L}_{h}$ is the reflexive hull of $\mathcal{L}_{M} \cup \mathcal{L}_{S}$.

Proof. Part (i) is elementary. Part (iii) is contained in the last proof and (ii) follows from (iii). 


\section{References}

[1] William Arveson. Operator algebras and invariant subspaces. Ann. of Math., 100(2):433-532, 1974.

[2] Kenneth R. Davidson. Nest algebras, volume 191 of Pitman Research Notes in Mathematics Series. Longman Scientific \& Technical, Harlow, 1988.

[3] John Froelich. Compact operators, invariant subspaces, and spectral synthesis. J. Funct. Anal., 81(1):1-37, 1988.

[4] A. Katavolos and S. C. Power. The Fourier binest algebra. Math. Proc. Camb. Phil. Soc., 122(3):525-539, 1997.

[5] A. Katavolos and S. C. Power. Translation and dilation invariant subspaces of $L^{2}(\mathbb{R})$. J. Reine Angew. Math., 552:101-129, 2002.

[6] Jon Kraus. The slice map problem and approximation properties. J. Funct. Anal., 102(1):116-155, 1991.

[7] David R. Larson and Warren R. Wogen. Reflexivity properties of $T \oplus 0$. J. Funct. Anal., 92(2):448-467, 1990.

[8] S. C. Power. Completely contractive representations for some doubly generated antisymmetric operator algebras. Proc. Amer. Math. Soc., 126(8):2355-2359, 1998.

[9] Donald Sarason. Weak-star density of polynomials. J. Reine Angew. Math., 252:1-15, 1972.

[10] James E. Thomson. Invariant subspaces for subnormal operators. In John B. Conway and Bernard B. Morrel, editors, Surveys of some recent results in operator theory, Vol. I, volume 171 of Pitman Research Notes in Mathematics Series, pages 241-259. Longman Scientific \& Technical, Harlow, 1988.

[11] W. R. Wogen. Some counterexamples in nonselfadjoint algebras. Ann. of Math., 126(2):415-427, 1987.

Department of Mathematics and Statistics, Lancaster University, LA1 4YF, ENGLAND

Fax: +44 1524592681

E-mail addresses: r.levene@lancaster.ac.uk, s.power@lancaster.ac.uk 